Nonlin. Processes Geophys., 17, 201-210, 2010

www.nonlin-processes-geophys.net/17/201/2010/

(C) Author(s) 2010. This work is distributed under

the Creative Commons Attribution 3.0 License.

\title{
The effect of shear on the generation of gravity waves
}

\author{
M. Humi \\ Department of Mathematical Sciences, Worcester Polytechnic Institute, 100 Institute Road, Worcester, MA 01609, USA
}

Received: 2 December 2009 - Revised: 23 March 2010 - Accepted: 25 March 2010 - Published: 12 April 2010

\begin{abstract}
Previous research regarding the solutions of Long's equation always presumed that the flow far upstream is without shear. In this paper we derive the proper form of this equation when shear is present. We then apply a sequence of transformations to this equation which make it possible to linearize it while preserving its physical contents. We then derive conditions under which the solutions of this linear equation admit the existence (or creation) of gravity waves. We present also a solution of this model equation when the presence of shear in the overall flow is "small".
\end{abstract}

\section{Introduction}

Long's equation (Long, 1952, 1953, 1955, 1959) models the flow of stratified incompressible fluid in two dimensions over terrain. When the base state of the flow (that is the unperturbed flow field far upstream) is without shear the numerical solutions (in the form of steady lee waves) of this equation over simple topography (i.e. one hill) were studied by many authors (Drazin, 1961, 1967; Durran, 1992; Lily, 1979; Peltier and Clarke, 1983; Smith, 1980, 1989, Yih 1967, Davis 1999). The most common approximation in these studies was to set Brunt-Väisälä frequency to a constant or a step function over the computational domain. Moreover the values of two physical parameters which appear in this equation were set to zero. (These parameters control the stratification and dispersive effects of the atmosphere see Sect. 2.) In this (singular) limit the nonlinear terms and one of the leading second order derivatives in the equation drop out and the equation reduces to that of a linear harmonic oscillator over two dimensional domain. Careful studies (Lily, 1979) showed that these approximations set strong limitations on the validity of the derived solutions (Peltier and Clarke, 1983). An extensive list of references appears in (Baines, 1995; Carmen, 2002; Yih, 1980).

Correspondence to: M. Humi (mhumi@wpi.edu)
Long's equation also provides the theoretical framework for the analysis of experimental data (Shutts, 1988, 1994; Vernin, 2007) under the assumption of shearless base flow. (An assumption which, in general, is not supported by the data; Humi, 2004b).

An analytic approach to the study of the solutions of this nonlinear equation was initiated recently by the current author (Humi, 2004a, 2006, 2007). We showed that for a base flow without shear and under rather mild restrictions the nonlinear terms in the equation can be simplified. Using phase averaging approximation we derived for self similar solutions of this equation a formula for the attenuation of the stream function perturbation with height. This result is generically related to the presence of the nonlinear terms in Long's equation. New representations of this equation in terms of the atmospheric density and terrain following coordinates were derived in (Humi, 2007, 2009). Partial results on the impact that shear can have on the generation and amplitude of gravity waves when the base flow consists of "pure shear" were investigated by us in (Humi, 2006).

The objective of this paper is to study the nature of the solutions to Long's equation when some shear is present in the base flow. Using conditions which depend solely on the base flow and Brunt-Väisälä frequency we characterize the qualitative nature of the perturbations from the base flow and their amplitude with height. These results are independent of the actual detailed description of the terrain that caused these perturbations. Furthermore we derive conditions under which these perturbations are not oscillatory i.e. no gravity waves are generated by the flow. To our best knowledge this issue was never considered in the literature before (at least in the context of Long's equation).

From a geophysical point of view it well known that some present models for the generation of gravity waves over estimate this effect (Eckermann and Preusse, 1999; Dewan et al., 1998; Doyle et al., 2006). Partially, this is due to the fact that shear is not taken into account, the representation of the terrain is oversimplified or some other crude (geophysical) assumptions are made.

Published by Copernicus Publications on behalf of the European Geosciences Union and the American Geophysical Union. 
The plan of the paper is as follows: Sect. 2 presents the different forms of Long's equation and the solution of its simplified version without shear. In Sect. 3 we derive the (analytic) solution of this simplified equation using terrain following formulation of Long's equation in the presence of shear. Section 4 presents several transformations of Long's equation which lead to it linearization while conserving its physical contents. We then solve this linearized equation when the mixture of shear in overall base flow is "small". Section 5 presents constraints on the generation of gravity waves in the presence of shear using the linearized equation which was derived in Sect. 4. Setion 6 considers the case of base flow consisting of "pure shear". We end up in Sect. 7 with summary and conclusions.

\section{Long's equation}

\subsection{Derivation of the equation}

In two dimensions $(x, z)$ the flow of a steady inviscid and incompressible stratified fluid (in the Boussinesq approximation) is modeled by the following equations:

$u_{x}+w_{z}=0$

$u \rho_{x}+w \rho_{z}=0$

$\rho\left(u u_{x}+w u_{z}\right)=-p_{x}$

$\rho\left(u w_{x}+w w_{z}\right)=-p_{z}-\rho g$

where subscripts indicate differentiation with respect to the indicated variable, $u=(u, w)$ is the fluid velocity, $\rho$ is its density $p$ is the pressure and $g$ is the acceleration of gravity.

We can non-dimensionalize these equations by introducing

$\bar{x}=\frac{x}{L}, \bar{z}=\frac{N_{0}}{U_{0}} z, \bar{u}=\frac{u}{U_{0}}, \bar{w}=\frac{L N_{0}}{U_{0}^{2}} w$

$\bar{\rho}=\frac{\rho}{\rho_{0}}, \bar{p}=\frac{N_{0}}{g U_{0} \rho_{0}} p$

where $L$ represents a characteristic length, and $U_{0}, \rho_{0}$ represent respectively the free stream velocity and density. $N_{0}$ is the characteristic Brunt-Vaisala frequency

$N_{0}^{2}=-\frac{g}{\rho_{0}} \frac{d \rho_{0}}{d z}$.

In these new variables Eqs. (1)-(4) take the following form (for brevity we drop the bars)

$$
\begin{aligned}
& u_{x}+w_{z}=0 \\
& u \rho_{x}+w \rho_{z}=0 \\
& \beta \rho\left(u u_{x}+w u_{z}\right)=-p_{z} \\
& \beta \rho\left(u w_{x}+w w_{z}\right)=-\mu^{-2}\left(p_{z}+\rho\right)
\end{aligned}
$$

where

$$
\begin{aligned}
& \beta=\frac{N_{0} U_{0}}{g} \\
& \mu=\frac{U_{0}}{N_{0} L} .
\end{aligned}
$$

$\beta$ is the Boussinesq parameter (Baines, 1995; Carmen, 2002) which controls stratification effects (assuming $U_{0} \neq 0$ ) and $\mu$ is the long wave parameter which controls dispersive effects (or the deviation from the hydrostatic approximation). In the limit $\mu=0$ the hydrostatic approximation is fully satisfied (Baines, 1995; Carmen, 2002).

In view of Eq. (7) we can introduce a stream function $\psi$ so that

$u=\psi_{z}, w=-\psi_{x}$.

After a long (and intricate) algebra one can show that $\rho=\rho(\psi)$ and derive the following equation for $\psi$ (Dubreil, 1934; Long, 1953, 1954)

$\psi_{z z}+\mu^{2} \psi_{x x}-N^{2}(\psi)\left[z+\frac{\beta}{2}\left(\psi_{z}^{2}+\mu^{2} \psi_{x}^{2}\right)\right]=S(\psi)$

where

$N^{2}(\psi)=-\frac{\rho_{\psi}}{\beta \rho}$

is the nondimensional Brunt-Vaisala frequency and $S(\psi)$ is some unknown function which can be determined by making proper assumptions on the upstream disturbance (see Baines, 1995; Carmen, 2002). Equation (14) is referred to as Long's equation. We note that a generalization of this equation in the context of ocean internal gravity waves appeared in the literature (Miropol'sky, 1974, 2001).

If we let

$\psi(-\infty, z)=z$

then

$S(\psi)=-N^{2}(\psi)\left(\psi+\frac{\beta}{2}\right)$

and Eq. (14) becomes:

$\psi_{z z}+\mu^{2} \psi_{x x}-N^{2}(\psi)\left[z-\psi+\frac{\beta}{2}\left(\psi_{z}^{2}+\mu^{2} \psi_{x}^{2}-1\right)\right]=0$.

On the other hand if we include shear in the base flow viz we let

$\psi(-\infty, z)=z+\frac{\delta}{2} z^{2}, \delta \geq 0$,

then

$S(\psi)=\delta-N^{2}(\psi)\left[\frac{\sqrt{1+2 \delta \psi}-1}{\delta}+\frac{\beta}{2}(1+2 \delta \psi)\right]$ 
and Long's equation takes the following form;

$$
\begin{aligned}
& \psi_{z z}+\mu^{2} \psi_{x x}-N^{2}(\psi)\left[z+\frac{\beta}{2}\left(\psi_{z}^{2}+\mu^{2} \psi_{x}^{2}\right)\right] \\
& =\delta-N^{2}(\psi)\left[\frac{\sqrt{1+2 \delta \psi}-1}{\delta}+\frac{\beta}{2}(1+2 \delta \psi)\right] .
\end{aligned}
$$

When $\delta \ll 1$, we can approximate this equation by

$$
\begin{aligned}
& \psi_{z z}+\mu^{2} \psi_{x x}-N^{2}(\psi)\left[z+\frac{\beta}{2}\left(\psi_{z}^{2}+\mu^{2} \psi_{x}^{2}\right)\right] \\
& =\delta-N^{2}(\psi)\left[\psi+\frac{\beta}{2}(1+2 \delta \psi)\right] .
\end{aligned}
$$

\subsection{Terrain following formulation}

Recently a terrain following formulation of Long's equation was derived in the literature. In this formulation one introduces a transformation of the coordinates

$\bar{x}=x, \quad \bar{z}=H \frac{z-h(x)}{H-h(x)}$

where $h(x)$ is the height of the terrain and $H$ is the vertical scale of the domain. Under this transformation we have

$$
\frac{\partial}{\partial x}=\frac{\partial}{\partial \bar{x}}+G^{12} \frac{\partial}{\partial \bar{z}}, \frac{\partial}{\partial z}=\frac{1}{\sqrt{G}} \frac{\partial}{\partial \bar{z}}
$$

where

$$
\frac{1}{\sqrt{G}}=\frac{H}{H-h(x)}, G^{12}=\frac{1}{\sqrt{G}}\left(\frac{\bar{z}}{H}-1\right) h^{\prime}(x) .
$$

Next a "terrain following stream function" is defined by the following relations,

$\bar{u}=\sqrt{G} u=\frac{\partial \psi}{\partial \bar{z}}, \quad \bar{v}=\sqrt{G} v=-\frac{\partial \psi}{\partial \bar{x}}$.

In this formulation Long's equation takes the following form

$\bar{\nabla}_{\mu}^{2} \psi-\frac{N^{2}(\psi) \beta}{2}\left[\mu^{2}\left(\psi_{\bar{x}}\right)^{2}+2 \mu^{2} G^{12} \psi_{\bar{x}} \psi_{\bar{z}}\right.$

$\left.+\left(\frac{1}{G}+\mu^{2}\left(G^{12}\right)^{2}\right)\left(\psi_{\bar{z}}\right)^{2}\right]-N^{2}(\psi) g(\bar{x}, \bar{z})=S(\psi)$.

where

$\bar{\nabla}_{\mu}^{2}=\mu^{2}\left\{\frac{\partial^{2}}{\partial \bar{x}^{2}}+2 G^{12} \frac{\partial^{2}}{\partial \bar{x} \partial \bar{z}}+\left[\frac{\partial G^{12}}{\partial \bar{x}}+G^{12} \frac{\partial G^{12}}{\partial \bar{z}}\right] \frac{\partial}{\partial \bar{z}}\right\}$

$+\left[\frac{1}{G}+\mu^{2}\left(G^{12}\right)^{2}\right] \frac{\partial^{2}}{\partial \bar{z}^{2}}$

and

$g(\bar{x}, \bar{z})=\bar{z}+h(\bar{x})\left(1-\frac{\bar{z}}{H}\right)$.

When the base flow is shearless i.e. satisfies Eq. (16) $S(\psi)$ in (27) is given by (17). Similarly if the base flow satisfies (19) then $S(\psi)$ is given by (20).

\subsection{Equations for the perturbation from the base flow}

For the perturbation $\eta$ from shearless base flow (16) we have

$\eta=\psi-z$.

Equation (18) becomes

$\eta_{z z}-\alpha^{2} \eta_{z}^{2}+\mu^{2}\left(\eta_{x x}-\alpha^{2} \eta_{x}^{2}\right)-N^{2}(\eta)\left(\beta \eta_{z}-\eta\right)=0$

where

$\alpha^{2}=\frac{N^{2}(\psi) \beta}{2}$.

Similarly to derive an equation for the perturbation $\phi$ from the shear base flow (19), we let

$\eta=\psi-z-\frac{\delta}{2} z^{2}$.

Substituting this in (21) we obtain

$\eta_{z z}-\alpha^{2} \eta_{z}^{2}+\mu^{2}\left(\eta_{x x}-\alpha^{2} \eta_{x}^{2}\right)-2 \alpha^{2}\left[(1+\delta z) \eta_{z}-\delta \eta\right]$

$-N^{2}\left[z+\frac{1}{\delta}\left(1-\sqrt{(1+\delta z)^{2}+2 \delta \eta}\right)\right]=0$

Observe that both (30) and (33) are exact equations for $\eta$. However, if $|\delta \eta| \ll 1$ we can remove $1+\delta z$ from the square root in (33) and use the approximation $\sqrt{1+a} \approx 1+a / 2$ to obtain

$\eta_{z z}-\alpha^{2} \eta_{z}^{2}+\mu^{2}\left(\eta_{x x}-\alpha^{2} \eta_{x}^{2}\right)-2 \alpha^{2}\left[(1+\delta z) \eta_{z}-\delta \eta\right]$

$+\frac{N^{2} \eta}{1+\delta z}=0$

\subsection{Boundary conditions}

For a shearless flow in an unbounded domain over topography with shape $f(x)$ and maximum height $h_{\max }$ the following boundary conditions are imposed on $\psi$

$\psi(-\infty, z)=z$

$\psi(x, \epsilon f(x))=$ constant,$\epsilon=\frac{h_{\max } N_{0}}{U_{0}}$

where the constant in Eq. (36) is (usually) set to zero. For low lying topography (viz $\epsilon \ll 1$ ) it is customary to replace (in the numerical simulations) (36) by the approximation

$\eta(x, 0)=-\epsilon f(x)$.

As to the boundary condition on $\psi(\infty, z)$ we observe that Long's equation contains no dissipation terms and therefore only radiation boundary conditions can be imposed in this limit. Similarly at $z=\infty$ it is customary to impose (following Lily, 1979; Durran, 1992) radiation boundary conditions. When shear is present in the base flow (35) is replaced by (19). 
In the terrain following formulation of Long's equation the boundary condition (36) is replaced by

$\psi(\bar{x}, 0)=0$

where $\psi$ is the terrain following stream function.

For the rest of this paper we consider this equation for the special case where $N$ and $\delta$ are constants.

\section{The limiting case $\beta=0, \mu=0$}

When $\beta=0, \mu=0$ and $N(\psi)$ is a constant $N$ over the domain Eq. (30) reduces to a linear equation

$\eta_{z z}+N^{2} \eta=0$

We observe that the limit $\beta=0$ can be obtained either by letting $U_{0} \rightarrow 0$ or $N_{0} \rightarrow 0$. In the following we assume that this limit is obtained as $U_{0} \rightarrow 0$ (so that stratification persists in this limit). The general solution of Eq. (39) is

$\eta(x, z)=p(x) \cos (N z)+q(x) \sin (N z)$

where the functions $p(x), q(x)$ have to be chosen so that the the boundary conditions derived from Eq. (35), (36) and the radiation boundary conditions are satisfied. These lead in general to an integral equation for $p(x)$ and $q(x)$.

$q(x) \cos (\epsilon N f(x))+H[q(x)] \sin (\epsilon N f(x))=-\epsilon f(x)$.

where $H[q(x)]$ is the Hilbert transform of $q(x)$. This equation has to be solved numerically (Davis, 1999; Drazin, 1961, 1969) subject to the boundary conditions mentioned above. However recently (Humi, 2009) we showed how this problem can be solved analytically using a "terrain following formulation" of Long's equation.

It is clear from the form of the general solution given by Eq. (40) that it represents a wave propagating in the $\mathrm{z}$ direction and the properties of this wave (under varied physical conditions) were investigated by the authors which were mentioned in Sect. 1. It should be observed however that Eq. (39) is a "singular limit" of Long's equation as one of the leading second order derivatives drops when $\mu=0$ and the nonlinear terms drop when $\beta=0$. Under these circumstances it is uncertain that the solutions of "limit equation" relates continuously to the solutions of the original equation. It is imperative therefore to investigate other forms of Eq. (18) (or equivalently Eq. 30) and explore the impact of these "limit approximations" on the solution.

Under the same limits mentioned above (34) reduces to

$\eta_{z z}+N^{2} \eta=-\frac{N^{2} \delta}{2} z^{2}$

whose general solution is

$\eta(x, z)=p(x) \cos (N z)+q(x) \sin (N z)-\delta\left(z^{2}-\frac{2}{N^{2}}\right)$.
Observe that when $\delta=0$ Eq. (43) reduces to (40), i.e. the extra term in (43) is due to the presence of shear.

Applying the boundary conditions to this solution (41) is replaced by

$q(x) \cos (\epsilon N f(x))+H[q(x)] \sin (\epsilon N f(x))=-\epsilon f(x)$

$+\delta\left(\epsilon^{2} N^{4} f(x)^{2}-\frac{2}{N^{2}}\right)$.

In the terrain following formulation of Long's equation the corresponding equation for the terrain following stream function in these limits with $\delta \ll 1$ is

$\psi_{\bar{z} \bar{z}}+G N^{2} \psi=G\left\{\delta+N^{2}\left[\bar{z}+h(\bar{x})\left(1-\frac{\bar{z}}{H}\right)\right]\right\}$

The general solution of this equation is

$\psi=A(\bar{x}) \cos (v \bar{z})+B(\bar{x}) \sin (v \bar{z})+\left[\bar{z}+h(\bar{x})\left(1-\frac{\bar{z}}{H}\right)\right]+\frac{\delta}{N^{2}}$.

where $h(x)=\epsilon f(x)$ and $v^{2}=G N^{2}$. Applying the boundary conditions at $\bar{z}=0$ yields $A(\bar{x})=-h(\bar{x})-\frac{\delta}{N^{2}}$. Using the radiation boundary conditions implies that $B(\bar{x})=H(A(\bar{x}))$ (see Humi, 2009 for a detailed discussion of this derivation).

If $f(x)$ is given by a "witch of Agnesi" curve

$f(x)=\frac{a^{2}}{\left(a^{2}+x^{2}\right)}$

then

$B(x)=-\epsilon \frac{a x}{a^{2}+x^{2}}$.

Here we used the fact that the Hilbert transform of a constant is zero and $\bar{x}=x$. For comparison purpose Figs. 1 and 2 present the solutions of Eq. (45) with and without shear over this topography. We observe that in Eq. (46) $v^{2}=G N^{2}$ is not constant since $G$ is a function of $x$. However, in the region where $h(x)=0, G=1$ and therefore $v=N$. For Fig. 1, $N=$ 1 and since $\delta=0$ it follows that the base flow speed $U=1$, hence asymptotically (i.e. as $x \rightarrow \infty$ ) $v=1$. In Fig. 2, $U$ is not constant with height and is equal to $U=1+\delta z$; see (19). Hence the Richardson number for this flow is $R i=$ $N^{2} / U_{z}^{2}=N^{2} / \delta^{2}$. In Fig. $2, N=1$ and $\delta=0.1$.

\section{Transformations on Long's equation with shear}

Long's equation in the form (22) contains second order derivatives in $x, z$ and quadratic terms in the first order derivatives. In this form it is a challenge to solve the full equation even numerically. In this section we apply on this equation a sequence of transformations which mitigate some of these difficulties. 


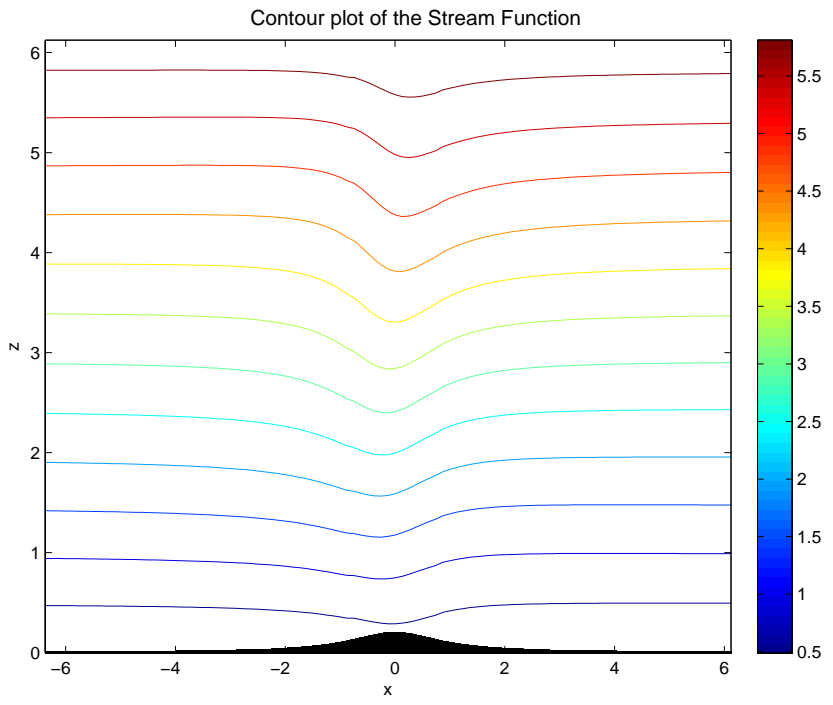

Fig. 1. Solution of Eq. (45) over a "witch of Agnesi" topography with $\epsilon=0.2, \delta=0, N=1, \beta=\mu=0$.

To begin with we define $\bar{z}=z-\beta / 2+\delta / N^{2}, \bar{x}=x / \mu$ and substitute in Eq. (22) we obtain after dropping the bars that

$$
\left(\psi_{z z}-\alpha^{2} \psi_{z}^{2}\right)+\left(\psi_{x x}-\alpha^{2} \psi_{x}^{2}\right)+N^{2}(\psi)[(1+\beta \delta) \psi-z]=0
$$

Assuming that $N^{2}(\psi)$ is constant we now apply the transformation

$\phi=e^{-\alpha^{2} \psi}, \alpha \neq 0$.

Equation (49) becomes

$\nabla^{2} \phi+N^{2} \phi\left(\alpha^{2} z+(1+\beta \delta) \ln \phi\right)=0$.

Similar transformations can be applied to the perturbation equation (34). In fact if we let $\bar{x}=x / \mu$ and introduce

$\chi=e^{-\alpha^{2} \eta}, \alpha \neq 0$.

then (34) takes the form

$\nabla^{2} \chi-2 \alpha^{2}(1+\delta z) \chi_{z}+\left(2 \alpha^{2} \delta+\frac{N^{2}}{1+\delta z}\right) \chi \ln \chi=0$.

We observe that $\left|\alpha^{2} \eta\right| \ll 1$ therefore $\chi \approx 1$. For this reason we now introduce $p(x, z)=1-\chi(x, z)$ (so that $|p(x, z)| \ll 1$ again) and let $\ln (1+x) \approx x$ for $|x| \ll 1$. We obtain

$\nabla^{2} p-2 \alpha^{2}(1+\delta z) p_{z}+\left(2 \delta \alpha^{2}+\frac{N^{2}}{1+\delta z}\right)\left(p-p^{2}\right)=0$.

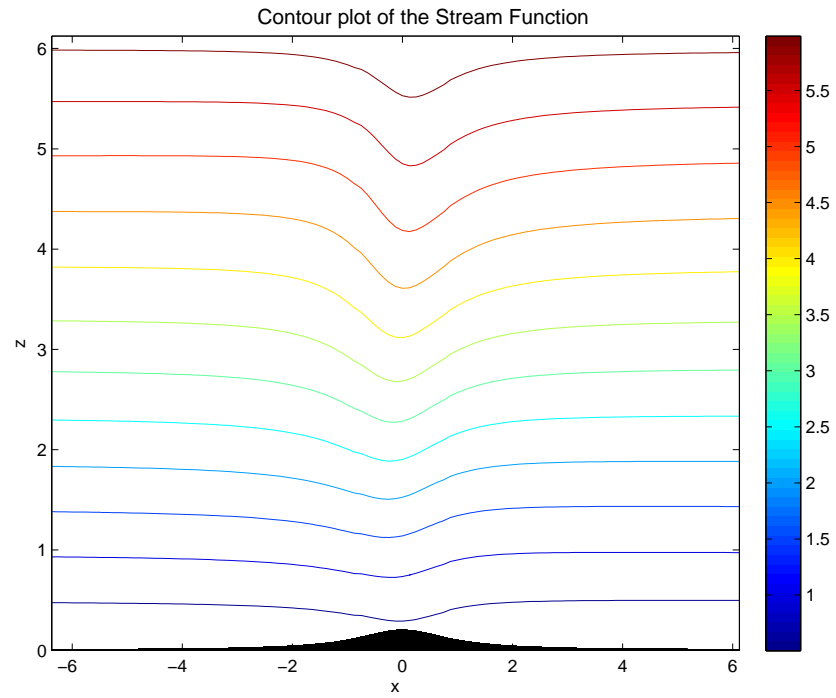

Fig. 2. Same as Fig. 1 with $\delta=0.1$.

However since $|p(x, z)| \ll 1$ we can neglect the nonlinear terms in this equation and obtain the following linear approximation to Long's equation.

$\nabla^{2} p-2 \alpha^{2}(1+\delta z) p_{z}+\left(2 \delta \alpha^{2}+\frac{N^{2}}{1+\delta z}\right) p=0$.

We observe that the only other approximation that was made in the derivation of this equation is to assume that $|\delta \eta| \ll$ 1. This equation is obviously superior to the usual linear approximation made in the literature with $\mu=0, \beta=0$ (and $\delta=0)$.

To solve (55) we apply separation of variables and let $p(x, z)=X(x) Z(z)$. After substitution we obtain

$X(x)^{\prime \prime}+k^{2} X(x)=0$,

$Z(z)^{\prime \prime}-2 \alpha^{2}(1+\delta z) Z(z)^{\prime}+\left[2 \delta \alpha^{2}+\frac{N^{2}}{1+\delta z}-k^{2}\right] Z(z)=0$

where $k$ is a (separation of variables) constant.

The solution to (56) is obvious. However the general solution to (57) can be expressed in terms elementary functions only if $\delta=0$. For other values of $\delta$ the general solution of this equation can be expressed in terms of Heun functions and their integrals. A representative plot of one of these solutions is shown in Fig. 3. We observe that the wave amplitude grows with height due to the effect of shear. If we plot this figure with the same parameters but with $\delta=0$ the amplitude remains almost constant (see next subsection). This 


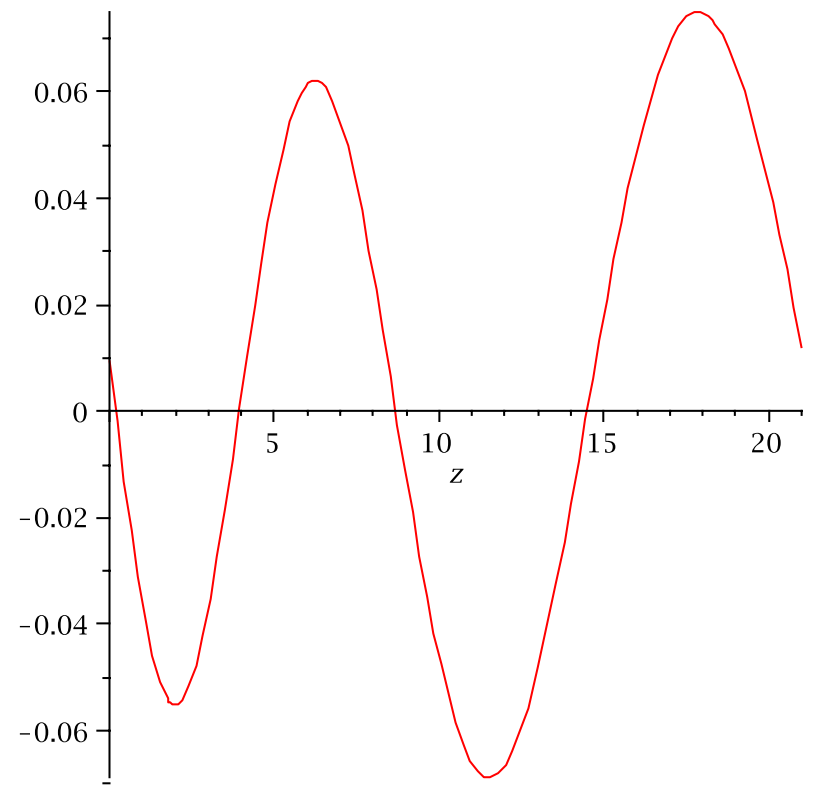

Fig. 3. A plot of of one of the solutions to (99) with $N=1, \delta=0.2$, $\alpha^{2}=0.0001$ and $\omega=0.1$.

plot was obtained using the numerical implementation of the Heun functions in MAPLE with a precision of 30 digits. The need to use this high precision stems from the nature of Heun functions and the roundoff errors in their evaluation (at lower precision).

\subsection{Solution for $\delta=0$}

When $\delta=0$ an oscillatory solution for $Z(z)$ in (57) exists when $v^{2}=N^{2}-\alpha^{4}-k^{2}>0$ and we have

$Z(z, k)=e^{\alpha^{2} z}\left[C_{1}(k) \cos v z+C_{2}(k) \sin v z\right]$

This solution shows that the linear theory of gravity waves which is based on Eq. (39) is valid only when the modulating factor $e^{\alpha^{2} z}$ is close to 1 , i.e. $\left|\alpha^{2} z\right| \ll 1$. It also shows how the wave amplitude is modulated by height due to effects of stratification. In addition we note that in the standard linear theory of gravity waves the condition for oscillatory solutions is $N^{2} / U^{2}-k^{2}>0$. This is due to the fact that this theory neglects the effect of stratification on the wave amplitude. (Note that in our discussion here $U=1$ when $\delta=0$ ). Equation (58) can be viewed therefore as a (minor) refinement of this theory.

In view of (58) the general solution for $p(x, z)$ can be written in the form

$p(x, z)=\int_{-\sqrt{N^{2}-\alpha^{4}}}^{\sqrt{N^{2}-\alpha^{4}}} e^{\alpha^{2} z}\left[D_{1}(k) \cos v z+D_{2}(k) \sin v z\right] e^{i k x} d k$ where $k$ is the horizontal wave number. To determine the constants $D_{1}(k), D_{2}(k)$ we have to apply the boundary condition to this solution. Since $\eta(x, 0)$ has to satisfy (37) it follows from (52) and the definition of $p(x, z)$ that

$p(x, 0)=1-e^{\epsilon f(x)}=H(x)$.

Hence

$D_{1}(k)=\frac{1}{2 \pi} \int_{-\infty}^{\infty} H(x) e^{-i k x} d x$.

To satisfy the radiation boundary condition as $z \rightarrow \infty$ we must insure that the vertical group velocity of the wave is positive. Using the dispersion relation for hydrostatic flow given in (Baines, 1995, p. 181) this group velocity is:

$c_{g}=\frac{N k \operatorname{sgn}(v)}{v^{2}}$.

It follows that the vertical group velocity is positive when $k v \geq 0$.

To impose this condition on the solution (59) we reexpress it in the form

$$
\begin{aligned}
& p(x, z)=\frac{1}{2} \int_{-\sqrt{N^{2}-\alpha^{4}}}^{\sqrt{N^{2}-\alpha^{4}}}\left[\left(D_{1}(k)-i D_{2}(k)\right) e^{i(k x+v z)}\right. \\
& \left.+\left(D_{1}(k)+i D_{2}(k)\right) e^{i(k x-v z)}\right] d k .
\end{aligned}
$$

The radiation boundary condition for $z \rightarrow \infty$ will be satisfied if the first and second integrals vanish for $k<0$ and $k>0$ respectively. This implies that

$D_{1}(k)=-i \operatorname{sgn}(k) D_{2}(k)$.

\subsection{Solutions for $\delta \ll 1$}

Although Eq. (57) can be solved in terms of Heun functions it is not straightforward to delineate from this representation of the solution it general properties and apply to it the proper boundary conditions when $\delta \neq 0$. In this section we shall use proper approximations to this equation when $|\delta z| \ll 1$ in order to obtain a representation of the solution in terms of elementary functions. When $|\delta z| \ll 1$ we can approximate $\frac{1}{1+\delta z}$ by $(1-\delta z)$ and Eq. (57) becomes

$$
\begin{aligned}
& Z(z, k)^{\prime \prime}-2 \alpha^{2}(1+\delta z) Z(z, k)^{\prime} \\
& +\left[2 \delta \alpha^{2}+N^{2}(1-\delta z)-k^{2}\right] Z(z, k)=0 .
\end{aligned}
$$

We note that the solution of this equation can be expressed in term of Kummar functions. However to derive an approximate representation of the solution in terms of elementary functions we have to use a first order regular perturbation expansion of $Z(z)$ in terms of $\delta$ viz we let

$$
Z(z, k)=Z_{0}(z, k)+\delta Z_{1}(z, k) .
$$


Substituting this expression in (63) we obtain for the zero and first orders in $\delta$

$$
\begin{aligned}
& Z_{0}(z, k)^{\prime \prime}-2 \alpha^{2} Z_{0}(z, k)^{\prime}+\left(N^{2}-k^{2}\right) Z_{0}(z, k)=0 \\
& Z_{1}(z, k)^{\prime \prime}-2 \alpha^{2} Z_{1}(z, k)^{\prime}+\left(N^{2}-k^{2}\right) Z_{1}(z, k) \\
& =\left(N^{2} z-2 \alpha^{2}\right) Z_{0}(z, k)+2 \alpha^{2} z Z_{0}(z, k)^{\prime}
\end{aligned}
$$

Equation (65) is the same as (57) when $\delta=0$ and therefore the solution for $Z_{0}(z, k)$ is given by (58). Substituting this solution in (66) we find that the general solution for $Z_{1}(z)$ is

$$
Z_{1}(z)=e^{\alpha^{2} z}\left[\left(C_{3}+w_{1}(z)\right) \cos v z+\left(C_{4}+w_{2}(z)\right) \sin v z\right]
$$

where

$$
\begin{aligned}
\bar{w}_{1}(z) & =\frac{1}{4 v}\left[2 C_{2} \alpha^{2} v-C_{1}\left(N^{2}+2 \alpha^{4}\right)\right] z^{2} \\
& +\frac{1}{8 v^{3}}\left\{2 v \left[\left(C_{2}\left(N^{2}+2 \alpha^{4}\right)+6 C_{1} \alpha^{2} v\right] z\right.\right. \\
& \left.+C_{1}\left(N^{2}+2 \alpha^{4}\right)-6 C_{2} \alpha^{2} v\right\} \\
\bar{w}_{2}(z) & =\frac{1}{4 v}\left[2 C_{1} \alpha^{2} v+C_{2}\left(N^{2}+2 \alpha^{4}\right)\right] z^{2} \\
& +\frac{1}{8 v^{3}}\left\{2 v\left[C_{1}\left(N^{2}+2 \alpha^{4}\right)-6 C_{2} \alpha^{2} v\right] z\right. \\
& \left.-6 C_{1} \alpha^{2} v-C_{2}\left(N^{2}+2 \alpha^{4}\right)\right\}
\end{aligned}
$$

The general solution for $p(x, z)$ can be written as

$$
\begin{aligned}
& p(x, z) \\
& =\int_{-\sqrt{N^{2}-\alpha^{4}}}^{\sqrt{N^{2}-\alpha^{4}}} e^{\alpha^{2} z} A(k)\left\{\left[C_{1}(k)+\delta\left(C_{3}(k)+\bar{w}_{1}(z)\right)\right] \cos v z d k\right. \\
& \left.+\left[C_{2}(k)+\delta\left(C_{4}(k)+\bar{w}_{2}(z)\right)\right] \sin v z\right\} e^{i k x}
\end{aligned}
$$

Introducing the lumped constants $D_{1}(k)=A(k) C_{1}(k)$, $D_{2}(k)=A(k) C_{2}(k), D_{3}(k)=A(k) C_{3}(k)$, $D_{4}(k)=A(k) C_{4}(k)$ this solution can be rewritten as

$$
\begin{aligned}
& p(x, z) \\
& =\int_{-\sqrt{N^{2}-\alpha^{4}}}^{\sqrt{N^{2}-\alpha^{4}}} e^{\alpha^{2} z}\left\{\left[D_{1}(k)+\delta\left(D_{3}(k)+w_{1}(z)\right)\right] \cos v z d k\right. \\
& \left.+\left[D_{2}(k)+\delta\left(D_{4}(k)+w_{2}(z)\right)\right] \sin v z\right\} e^{i k x} \\
& =p_{0}(x, z)+\delta p_{1}(x, z)
\end{aligned}
$$

where

$$
\begin{aligned}
w_{1}(z) & =\frac{1}{4 v}\left[2 D_{2} \alpha^{2} v-D_{1}\left(N^{2}+2 \alpha^{4}\right)\right] z^{2} \\
& +\frac{1}{8 v^{3}}\left\{2 v \left[\left(D_{2}\left(N^{2}+2 \alpha^{4}\right)+6 D_{1} \alpha^{2} v\right] z\right.\right. \\
& \left.+D_{1}\left(N^{2}+2 \alpha^{4}\right)-6 D_{2} \alpha^{2} v\right\}
\end{aligned}
$$

$$
\begin{aligned}
w_{2}(z) & =\frac{1}{4 v}\left[2 D_{1} \alpha^{2} v+D_{2}\left(N^{2}+2 \alpha^{4}\right)\right] z^{2} \\
& +\frac{1}{8 v^{3}}\left\{2 v\left[D_{1}\left(N^{2}+2 \alpha^{4}\right)-6 D_{2} \alpha^{2} v\right] z\right. \\
& \left.-6 D_{1} \alpha^{2} v-D_{2}\left(N^{2}+2 \alpha^{4}\right)\right\} .
\end{aligned}
$$

The constants $D_{1}(k), D_{2}(k)$, are determined by the same procedure used in the previous section. To determine $D_{3}$ we apply the boundary condition $p_{1}(x, 0)=0$ which implies that

$$
D_{3}(k)=-\frac{1}{8 v^{3}}\left[D_{1}(k)\left(N^{2}+2 \alpha^{4}\right)-6 D_{2}(k) \alpha^{2} v\right] \text {. }
$$

To determine $D_{4}$ we apply the radiation boundary condition to the wave part of $p_{1}(x, z)$. This yields,

$D_{3}(k)=-i \operatorname{sgn}(k) D_{4}(k)$.

\section{Gravity waves generation in the presence of shear}

To determine the effect of shear (viz $\delta \neq 0)$ on the solution for $Z(z)$ in general and its oscillatory nature we shall apply Sturm-Picone oscillation theorems (Sturm, 1836; Picone, 1909). To this end we write (57) in self-adjoint form

$$
\begin{aligned}
& \frac{d}{d z}\left[e^{-\alpha^{2}\left(z+\delta z^{2} / 2\right)} \frac{d Z}{d z}\right] \\
& +\left[2 \delta \alpha^{2}+\frac{N^{2}}{1+\delta z}-\omega^{2}\right] e^{-\alpha^{2}\left(z+\delta z^{2} / 2\right)} Z=0
\end{aligned}
$$

For second order linear differential equations in this form a variant of Sturm comparison theorem states the following:

Theorem (Sturm) Assume that on the interval $0 \leq z \leq$ $Z_{\text {max }}$ the functions $K$ and $G$ in the differential equation

$\frac{d}{d z}\left[K(z) \frac{d y(z)}{d z}\right]-G(z) y(z)=0$

satisfy the inequalities

$A_{1} \geq K(z) \geq A_{2}>0, B_{1} \geq G(z) \geq B_{2}$.

Then

1. If $B_{2}>0$ the solutions of (77) are non-oscillatory. 
2. If $B_{2}<0$ and

$-\frac{B_{2}}{A_{2}}<\frac{\pi^{2}}{Z_{\max }^{2}}$

then the solutions of (77) are non-oscillatory.

3. A sufficient condition for the solutions of (77) to be oscillatory with at least $m$ zeroes on $0 \leq z \leq Z_{\max }$ is

$$
-\frac{B_{1}}{A_{1}} \geq \frac{m^{2} \pi^{2}}{Z_{\max }^{2}}
$$

For (76)

$K(z)=e^{-\alpha^{2}\left(z+\delta z^{2} / 2\right)}$

and

$G(z)=-\left[2 \delta \alpha^{2}+\frac{N^{2}}{1+\delta z}-\omega^{2}\right] e^{-\alpha^{2}\left(z+\delta z^{2} / 2\right)}$

It is obvious that we can choose $A_{1}=1$ and $A_{2}=$ $e^{-\alpha^{2}\left(Z_{\max }+\delta Z_{\max }^{2} / 2\right)}$. To find $B_{1}, B_{2}$ we rewrite the second inequality in (78) as

$-B_{1} \leq-G(z) \leq-B_{2}$.

It is easy to see then that a proper choice of these constants is

$B_{1}=-\left[2 \delta \alpha^{2}+\frac{N^{2}}{\left(1+\delta Z_{\max }\right)}-\omega^{2}\right] e^{-\alpha^{2}\left(Z_{\max }+\delta Z_{\max }^{2} / 2\right)}$

$B_{2}=-\left(N^{2}+2 \delta \alpha^{2}-\omega^{2}\right)$

From the first item in the list above we obtain that the solution is non-oscillatory if

$\left(N^{2}+2 \delta \alpha^{2}-\omega^{2}\right)<0$

From (79) we obtain that the solution is non-oscillatory if

$0<\left(N^{2}+2 \delta \alpha^{2}-\omega^{2}\right)<\frac{\pi^{2}}{Z_{\max }^{2}} e^{-\alpha^{2}\left(Z_{\max }+\delta Z_{\max }^{2} / 2\right)}$

Finally a sufficient condition for the solutions to oscillate and have at least $m$ zeroes is

$\left[\frac{N^{2}}{\left(1+\delta Z_{\max }\right)}+2 \delta \alpha^{2}-\omega^{2}\right] e^{-\alpha^{2}\left(Z_{\max }+\delta Z_{\max }^{2} / 2\right)}>\frac{m^{2} \pi^{2}}{Z_{\max }^{2}}$

We conclude then that a sufficient condition for oscillation to exist on the interval $\left[0, Z_{\max }\right]$ is that

$\left[\frac{N^{2}}{\left(1+\delta Z_{\max }\right)}+2 \delta \alpha^{2}\right]>\frac{\pi^{2}}{Z_{\max }^{2}} e^{\alpha^{2}\left(Z_{\max }+\delta Z_{\max }^{2} / 2\right)}$.
$Z(z)$ is not oscillatory if

$N^{2}+2 \delta \alpha^{2}<\frac{\pi^{2}}{Z_{\max }^{2}} e^{-\alpha^{2}\left(Z_{\max }+\delta Z_{\max }^{2} / 2\right)}$.

We infer therefore that the existence of shear will dampen the possible existence (or creation) of gravity waves since it main effect will be to decrease the value of the left hand side in (89) and increase the value of the right hand side. We observe that these results are valid for any solution of (57) and therefore especially for the solution that satisfies the boundary conditions.

As a specific illustration of the possible effect of shear on the generation of gravity waves we consider the inequality (89) for $N=0.4, \beta=0.025$ with $\delta=0$ (no shear) and $\delta=$ 0.37 as a function of $Z_{\max }$ (these values of the parameters are relevant in atmospheric studies). In Figs. 4 and 5 we plotted the values of the left hand side of the inequality (solid line) versus the values of the right side (dotted line) for these two values of $\delta$. We see that when no shear is present the inequality (89) is satisfied for $Z_{\max }>8$ but it is not satisfied for any value of $Z_{\max }$ for the case with shear. We infer then that under these circumstances gravity waves will be present if the base flow is shearless but no such waves will be present if the base flow contains a strong enough shear component. This result is in line with other investigations on the effect of shear on gravity waves in the atmosphere (Shutts, 2006; Dewan, 1998).

\section{Pure shear flow}

In the previous sections we considered a base flow which satisfies (19) with $0 \leq \delta \ll 1$. In this section we consider the "pure shear" case where

$\psi(-\infty, z)=z^{2}$.

In this case $u(-\infty, z)=z$ that is $u$ increases linearly with height. Using (14) we find that

$S(\psi)=2-N^{2}(\psi)\left[\psi^{1 / 2}+2 \beta \psi\right]$

and Long's equation (14) for $\psi$ becomes

$\left(\psi_{z z}-\alpha^{2} \psi_{z}^{2}\right)+\mu^{2}\left(\psi_{x x}-\alpha^{2} \psi_{x}^{2}\right)-N^{2}(\psi) z$

$=2-N^{2}(\psi)\left[\psi^{1 / 2}+2 \beta \psi\right]$.

To derive an equation for a perturbation from the base flow we set $\psi=z^{2}+\eta(x, z)$ and substitute in (93)

$\left(\eta_{z z}-\alpha^{2} \eta_{z}^{2}\right)+\mu^{2}\left(\eta_{x x}-\alpha^{2} \eta_{x}^{2}\right)-4 \alpha^{2}\left(z \eta_{z}-\eta\right)$

$-N^{2} z+N^{2} \sqrt{z^{2}+\eta}=0$. 


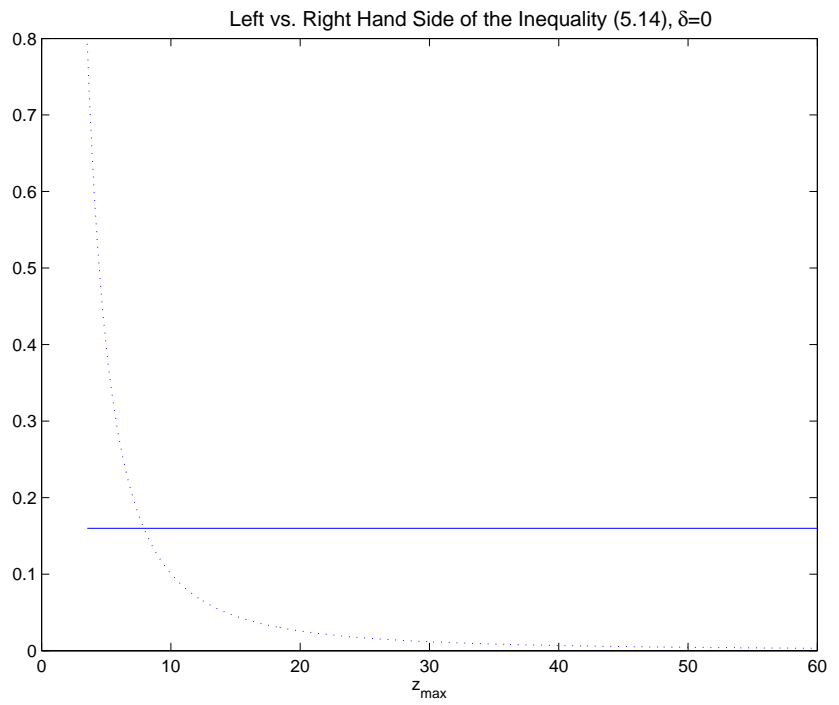

Fig. 4. A plot of the left hand of the inequality (89) (solid line) versus the right hand side (dotted line) when $N=0, \beta=0.025$ and $\delta=0$ as a function of $Z_{\max }$.

For $|\eta| \ll z$ we can approximate the square root in this equation by $\sqrt{z^{2}+\eta} \approx z+\eta /(2 z)$. This leads to

$$
\left(\eta_{z z}-\alpha^{2} \eta_{z}^{2}\right)+\mu^{2}\left(\eta_{x x}-\alpha^{2} \eta_{x}^{2}\right)-4 \alpha^{2}\left(z \eta_{z}-\eta\right)+\frac{N^{2}}{2 z} \eta=0
$$

On this equation we now apply the transformation $\bar{x}=x / \mu$ and then apply (52). We obtain

$\nabla^{2} \chi-4 \alpha^{2} z \chi_{z}+\left(\frac{N^{2}}{2 z}+4 \alpha^{2}\right) \chi \ln \chi=0$

Using the arguments which proceeded (54) and (55) we introduce $p(x, z)=1-\chi(x, z)$ and neglect the second order terms in $p$. This yields,

$\nabla^{2} p-4 \alpha^{2} z p_{z}+\left(\frac{N^{2}}{2 z}+4 \alpha^{2}\right) p=0$.

This equation can be solved by separation of variables. Introducing $p(x, z)=X(x) Z(z)$ we obtain for $X(x)$ (56) and for $Z(z)$ the following

$Z(z)^{\prime \prime}-4 \alpha^{2} z Z(z)^{\prime}+\left(\frac{N^{2}}{2 z}+4 \alpha^{2}-\omega^{2}\right) Z(z)=0$

The general solution of this equation can be expressed in term of Heun functions. To determine under what conditions this solution is oscillatory we rewrite (98) in the following form

$$
\frac{d}{d z}\left[e^{-2 \alpha^{2} z^{2}} \frac{d Z}{d z}\right]+\left(\frac{N^{2}}{2 z}+4 \alpha^{2} z-\omega^{2}\right) e^{-2 \alpha^{2} z^{2}} Z(z)=0
$$

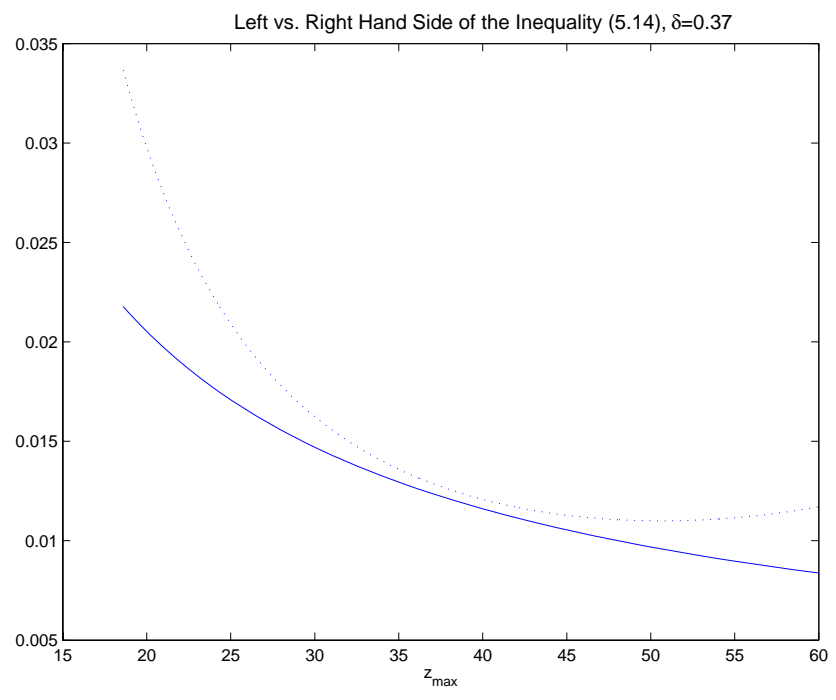

Fig. 5. Same as Fig. 4 with $\delta=0.37$.

and apply Sturm theorem on the interval $\left[z_{0}, Z_{\max }\right]$ (where $z_{0}$ is determined by the requirement that $\left.|\eta| \ll z_{0}\right)$. In this case $A_{1}=e^{-2 \alpha^{2} z_{0}^{2}}$ and $A_{2}=e^{-2 \alpha^{2} Z_{\max }^{2}}$. Similarly

$B_{1}=-\left(\frac{N^{2}}{2 Z_{\max }}+4 \alpha^{2} z_{0}-\omega^{2}\right) e^{-2 \alpha^{2} Z_{\max }^{2}}$

$B_{2}=-\left(\frac{N^{2}}{2 z_{0}}+4 \alpha^{2} Z_{\max }-\omega^{2}\right) e^{-2 \alpha^{2} z_{0}^{2}}$

We infer therefore that the solution will not be oscillatory if either

$\left(\frac{N^{2}}{2 z_{0}}+4 \alpha^{2} Z_{\max }-\omega^{2}\right)<0$,

or

$0<\left(\frac{N^{2}}{2 z_{0}}+4 \alpha^{2} Z_{\max }-\omega^{2}\right)<\frac{\pi^{2}}{Z_{\max }^{2}} e^{2 \alpha^{2}\left(z_{0}^{2}-Z_{\max }^{2}\right)}$.

Finally a sufficient condition for the solution to oscillate and has at least $m$ zeroes if

$\left(\frac{N^{2}}{2 Z_{\max }}+4 \alpha^{2} z_{0}-\omega^{2}\right) e^{-2 \alpha^{2} Z_{\max }^{2}}>\frac{m^{2} \pi^{2}}{Z_{\max }^{2}} e^{-2 \alpha^{2} z_{0}^{2}}$

We conclude then that a sufficient condition for an oscillation to exist on the interval $\left[z_{0}, Z_{\max }\right]$ is that

$\left(\frac{N^{2}}{2 Z_{\max }}+4 \alpha^{2} z_{0}\right)>\frac{\pi^{2}}{Z_{\max }^{2}} e^{2 \alpha^{2}\left(Z_{\max }^{2}-z_{0}^{2}\right)}$.

$Z(z)$ is not Oscillatory if

$\frac{N^{2}}{2 z_{0}}+4 \alpha^{2} Z_{\max }<\frac{\pi^{2}}{Z_{\max }^{2}} e^{2 \alpha^{2}\left(z_{0}^{2}-Z_{\max }^{2}\right)}$. 


\section{Summary and conclusions}

We derived in this paper the proper form of Long's equation with shear. Using a sequence of transformations and mild approximations which conserve the geophysical contents of this equation we were able to deduce some criteria for the excitation of gravity wave under these conditions. These criteria depend only the shear contents in the base flow, the value of $N^{2}$ and the stratification. These results will be useful both experimentally and theoretically. Currently the experimental practice is to ignore the shear in the base flow (Shutts et al., 1988; Vernin et al., 2007) and attempt to deduce the quantitative attributes of the gravity waves using the shearless Long's equation. As a result several models for the generation of gravity waves overestimate their production. These models can be refined now by taking this important feature into account.

Edited by: R. Grimshaw

Reviewed by: two anonymous referees

\section{References}

Baines, P. G.: Topographic effects in Stratified flows, Cambridge Univ. Press, New-York, 1995.

Nappo, C. J.: Atmospheric Gravity Waves, Academic Press, Boston, 2002

Davis, K. S.: Flow of Nonuniformly Stratified Fluid of Large Depth over Topography. M.Sc. thesis in Mechanical Engineering, MIT, Cambridge, MA, 1999.

Dewan, E. M., Picard, R. H., O’Neil, R. R., Gardiner, H. A., Gibson, J., Mill, J. D., Richards, E., Kendra, M., and Gallery, W. O.: MSX satellite observations of thunderstorm-generated gravity waves in mid-wave infrared images of the upper stratosphere, Geophys. Res. Lett., 25(7), 939-942, 1998.

Doyle, J. D., Volkert, H., Dornbrac, A., Hoinka, K. P., and Hogan, T. F.: Aircraft measurements and numerical simulations of mountain waves over the central Alps: A pre-MAP test case, Q. J. Roy. Meteor. Soc., 128, 2175-2184, 2006.

Drazin, P. G.: On the steady flow of a fluid of variable density past an obstacle, Tellus, 13, 239-251, 1961.

Drazin, P.G. and Moorem, D. W.: Steady two dimensional flow of fluid of variable density over an obstacle, J. Fluid. Mech., 28, 353-370, 1967.

Dubreil-Jacotin, M. L.: Sur la determination rigoureuse des ondes permanentes periodiques d'ampleur finie, J. Math. Pure. Appl., 13, 217-291, 1934.

Durran, D. R.: Two-Layer solutions to Long's equation for vertically propagating mountain waves, Q. J. Roy. Meteor. Soc., 118, 415-433, 1992

Eckermann, S. D. and Preusse, P.: Global measurements of stratospheric mountain waves from space, Science, 286, 1534-1537, 1999.
Humi, M.: On the Solution of Long's Equation Over Terrain, Il Nuovo Cimento C, 27, 219-229, 2004a.

Humi, M.: Estimation of Atmospheric Structure Constants from Airplane Data, J. Atmos. Ocean. Tech., 21, 495-500, 2004b.

Humi, M.: On the Solution of Long's Equation with Shear, Siam J. Appl. Math., 66(6), 1839-1852, 2006.

Humi, M.: Density representation of Long's equation, Nonlin. Processes Geophys., 14, 273-283, 2007, http://www.nonlin-processes-geophys.net/14/273/2007/.

Humi, M.: Long's equation in terrain following coordinates, Nonlin. Processes Geophys., 16, 533-541, 2009, http://www.nonlin-processes-geophys.net/16/533/2009/.

Lily, D. K. and Klemp, J. B.: The effect of terrain shape on nonlinear hydrostatic mountain waves, J. Fluid Mech., 95, 241-261, 1979.

Long, R. R.: Some aspects of the flow of stratified fluids I. Theoretical investigation, Tellus, 5, 42-57, 1953.

Long, R. R.: Some aspects of the flow of stratified fluids II. Theoretical investigation, Tellus, 6, 97-115, 1954.

Long, R. R.: Some aspects of the flow of stratified fluids III. Continuous density gradients, Tellus, 7, 341-357, 1955.

Long, R. R.: The motion of fluids with density stratification, J. Geophys. Res., 64, 2151-2163, 1959.

Miropol'sky, Yu. Z.: Propagation of internal waves in an ocean with horizontal density field non-uniformities, Izv. Atmos. Ocean. Phy+, 10, 312-318, 1974.

Miropol'sky, Yu. Z.: Dynamics of Internal Gravity waves in the Ocean, Kluwer, Boston, 2001.

Peltier, W. R. and Clark, T. L.: Nonlinear mountain waves in two and three spatial dimensions, Q. J. Roy. Meteor. Soc., 109, 527 548, 1983 .

Picone, M.: Sui valori eccezionali di un parametro da cui dipende un'equazione differenziale lineare del secondo ordine, Ann. Scuola Norm. Sup. Pisa, 11, 1-141, 1909 (in Italian).

Shutts, G. J., Kitchen, M., and Hoare, P. H.: A large amplitude gravity wave in the lower stratosphere detected by radiosonde, Q. J. Roy. Meteor. Soc., 114, 579-594, 1988.

Shutts, G. J., Healey, P., and Mobbs, S. D.: A multiple sounding technique for the study of gravity waves, Q. J. Roy. Meteor. Soc., 120, 59-77, 1994.

Shutts, G. J.: Stationary gravity-wave structure in flows with directional wind shear, Q. J. Roy. Meteor. Soc., 124, 1421-1442, 1998.

Smith, R. B.: Linear theory of stratified hydrostatic flow past an isolated mountain, Tellus, 32, 348-364, 1980.

Smith, R. B.: Hydrostatic airflow over mountains, Adv. Geophys., 31, 1-41, 1989.

Sturm, J. C. F.: Sur les equations differenielles lineares du second ordre, J. Math. Pure Appl., 1, 106-186, 1836 (in French).

Vernin, J., Trinquet, H., Jumper, G., Murphy, E., and Ratkowski, A.: OHP02 gravity wave campaign in relation to optical turbulence, Environ. Fluid Mech., 7, 371-382, 2007.

Yih, C.-S.: Equations governing steady two-dimensional large amplitude motion of a stratified fluid, J. Fluid Mech., 29, 539-544, 1967.

Yih, C.-S.: Stratified flows, Academic Press, New York, NY, 1980. 